\title{
Physiotherapy devices able to generate ethical dilemmas
}

\author{
Nadinne Roman* \\ Transilvania University of Braşov, Department of Basic, Prophylactic and Clinical Disciplines, \\ Nicolae Balcescu 56, Braşov, Romania
}

\begin{abstract}
Physical therapy is a medical specialty where the professionals help restore movement and function when someone is affected by injury, illness or disability. This paper wishes to establish the connection between ethics, physiotherapy and bioengineering. The research method was achieved using academic database searches based on specific keywords. A SWOT analysis of the physiotherapy devices utilization and design was made, for extracting ethical considerations. The main results suggest that physiotherapy devices are able to generate ethical dilemmas, classified in 4 main items: (1) Bioengineering in physical therapy, ethical and clinical standards for manufacturers; (2) Social impact of physical therapy devices and ethical issues; (3) Inter-professional lack of communication and ethical concerns; (4) Bioengineering ethical research and education. As conclusions, for the physical therapy or electrotherapy research equipment development, a multidisciplinary team is needed. The equipment used in rehabilitation must fulfil specific technical and scientific requirements drafted by the professionals.
\end{abstract}

\section{Introduction}

Physical therapy and psychiatry are medical specialties where the professionals helps restore movement and function when someone is affected by injury, illness or disability. A physiotherapist specialist provides medical health care services in a prophylactically manner for patients likely to develop musculoskeletal disorders. The physiatrists and physical therapists' activity are centered on patient's treatment personalization, seeking to improve their patient's quality of life. The rehabilitation area treats a broad scope of cases, from the brain and spinal cord injuries, to post traumatic sequelae, congenital locomotors disorders, and stroke and burn victims. Usually, these specialists coordinate a multidisciplinary team of professionals, like physical therapists, occupational therapists and collaborate with the specialized clinicians like neurologist, podiatrist, pediatrician, and other specialized doctors [1]

Physical therapy is a medical field where devices and forms of therapy used varies depending on several factors such as the type of disease, the affected tissue, body`s area or segment affected, the patient's age or other associated pathologies like respiratory diseases, cardiovascular diseases, blood coagulation, diabetes, rheumatoid or psychiatric disorders.

\footnotetext{
*Corresponding author: nadinneroman@gmail.com
} 
This paper wishes to establish the connection between ethics, physiotherapy, and bioengineering, considering the multifactorial elements involved in the field of physiotherapy.

\section{Method}

Research method was achieved using academic database searches and academic engines based on specific keywords like: "physiotherapy", "medical devices", "bioengineering", "ethics", "rehabilitation", "psychiatry", "moral", "electrotherapy", "bioethics". We developed search criteria for each database: Isi Web of Science, Scopus, Elsevier and Google Scholar, but the main papers resulted from one particular search engine-Google Scholar. The literature in this field is poor developed so the papers analyzed have heterogeneous structures. The main physiotherapy devices suppliers' user manuals were searched too.

We have analyzed the data from the papers and user's manuals selected performing a SWOT analysis for physiotherapy devices, highlighting their strengths, weaknesses, opportunities, and threats. After analyzing the weak and strong elements concerning physiotherapy and electrotherapy devices, we have extracted four major ethical considerations concerning physiotherapy devices.

\section{Results}

The main results suggest that physiotherapy devices are able to generate ethical dilemmas in design and use. The ethical issues were distributed in four items.

\subsection{Bioengineering in physical therapy, ethical and clinical standards for manufacturers}

Bioengineering is a multidisciplinary framework, with multiple application domains, starting with skin grafting, tissue replacement, bone reconstruction and further continuing with implantable devices, biomedical micro electro-mechanical systems, prosthetics or limb replacement, but also concerning neuro robotics or physical therapy and electrotherapy devices. With the expansion of the bioengineering field, it also occurred the approaches concerning ethical issues in this branch, technology that interact with human life made in order to solve problems in medicine and biology.

With the expansion of the bioengineering field also growth the concerning of ethical issues regarding technologies that interact with human life [2]. The robotics and bioengineering in physical therapy area are less developed comparing with industrial robotics. The robots' specification within these fields is different because the user interface is the human being compared to industrial robots which usually do not permit human interaction. Secondary, in rehabilitation medicine robots or devices, are often items used by patients and not necessarily by medical professionals [3]. The need for specific rules or legislation caused many institutions to create frameworks and guidelines for regulating the ethical standards concerning bioengineering development and human beings' safety. In this case, ethics is not only a philosophical branch, but it is practical and serves to human race beneficence [2].

For bioengineers is important to have ethical knowledge concerning device or materials manufacturing and to be able to make an ethical decision. An ethical dilemma arises when two major ethics principles are conflicting with each other. The practical aspects of ethics it is disputed in an engineering student's research. The research revealed that using case study interactive education, and implementing before the basic ethical information "plays a 
significant role in learning moral reasoning skills" [4]. There is a poor boundary line concerning the interface of physiotherapy medical devices towards the therapeutic or prophylaxis utilization. The manufacturers and the medical devices suppliers are developing new technologies and some of them can be used both on ill or healthy subjects. There are many forms of electrotherapy or physical therapy devices in which the end user is the simple patient or client. In this case, the user doesn't have the required knowledge to be able to assess if the devices use might harm. There are physiotherapy medical devices that are not recognized by insurance companies due to lack of sufficient clinical randomized trials background. In this scenario, it cannot be proved the manufacturer's device effectiveness or lack of contraindications. This is a major ethical concerning among the physiotherapy devices users, to apply a therapy which proves decades later to have harmful effects unknown at the right time [5].

A recent physical therapy device proves that usually is insufficient medical scientific study background in this field. A well-suited example is extracorporeal shock wave therapy (ESWT) which was developed almost 17 years ago, whose manufacturer user's guide does not specify the cavitation process through effects. A 2016 study proves that ESWT generates cavitation and produce side effects including blood vessel rupture, not mentioned in the device's patent or other medical suppliers' manufacturers [6-7].

Organizational and regulatory institutions like World Health Organization (WHO) and The European Economic Community have some regulations and guidelines concerning medical device approval.

One of WHO statements refer to medical devices safety and it argues for "shared responsibility among the stakeholders". There are no special conditions regarding physiotherapy devices or concerning the ethical aspects of bioengineering or medical devices manufacturing [8-9]. Ethical responsibilities should be included in patents and manufacturers activity, including those with a prophylactically purpose.

\subsection{Social impact of physical therapy devices and ethical issues}

Physical therapy devices and bioengineering products used in rehabilitation represent an interface between the physical therapists, doctors, engineers and patients [2]. The physical therapy equipment is produced and utilized with the scope to improve patient's quality of life and regain movement trough activities of daily living. Lately, the physical therapy industry and neuro robotics developed. The utilization of these devices is highly expensive and involves a lot of ethical challenges.

The educational level and performance of the physical therapists, the patient's education and rehabilitation emotional distress period are key factors in ethical issues emerged. Some of the physical therapists even asking themselves if their profession is in danger to disappear, according to the manufacturer and patents development. But shortly studying the robots' usefulness and interface, they proved to be only another instrument in the hands of physical therapists, having more the role to fulfill the neuromuscular disorders are more prevalent due to precarious lifestyles. And here comes a new ethical issue about how much such equipment (neuro robots or exoskeletons) justifies the costs of manufacturing and how much it actually reflects in the cost of equipment the idea of novelty. This kind of ethical problem balances the good of a small group of people with high financial standards, with frustration and poor health of poor people. The electrotherapy devices used in physical therapy are different types of electromagnetic waves, ultrasound, lasers, continuous current, high voltage currents, and low frequency or high-frequency currents. These types of electrical therapies are noninvasive treatment methods which may interfere each odder and cause patient's adverse effects. The ethical implications of manufacturing and using these devices is a gap in this field. The professional community should reconsider the ethics approach of these devices use and 
manufacture. The reconsiderations shall be applied during the prototyping and patenting phase and also after placing on the market.

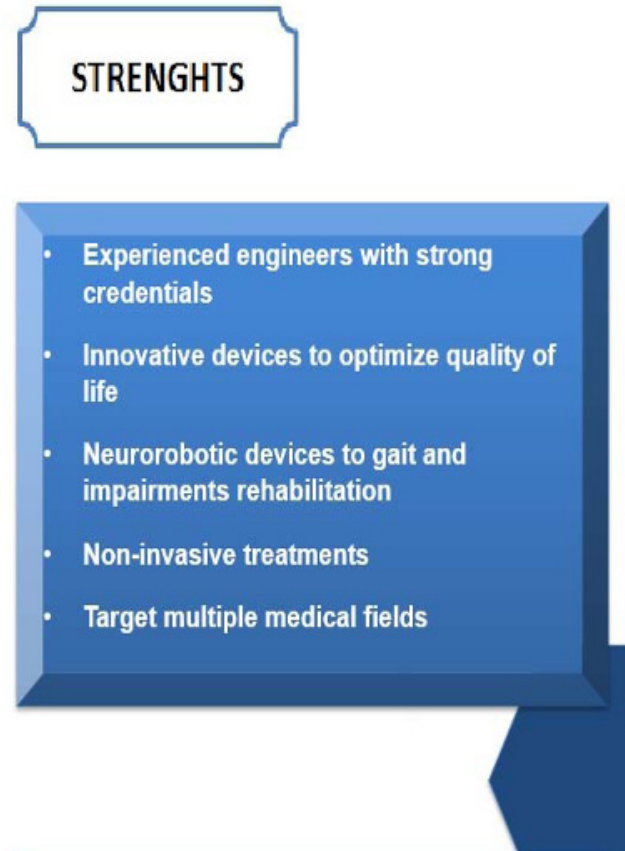

Market growth- expanded physiotherapy type of procedures

Increased emphasis on quality in healthcare

Growth the concerning of ethical issues regarding technologies that interact with human life

Ethical responsibilities included in patenting devices requirements

Governmental and institutionally ethics frameworks in design and use
Require expert help on developing and refining possible physiotherapy treatments Lack of interprofessional communication Lack of ethical standards for manufacturers Heterogeneous illness elements determine difficult to obtain accurate results

Poor ethical education concerning bioengineering

- Lack of ethical implications of manufacturing and use

High costs of new therapies, especially in public health care

Insufficient medical scientific study background

- Conflict of interests between bioengineers and manufacturers

Lack of applied ethics education

\section{Unshared responsibility}

\section{OPPORTUNITIES}

\section{THREATS}

Fig. 1. SWOT analysis for physiotherapy devices.

motor rehabilitation, not the functional recovery of the patients, with the advocacy for the inability of robots to understand the patient's needs and the proprioceptive feedback considered superior to visual feed-back [10]. 
And in terms of costs, physical therapy equipment reported at the level of neuro robots and exoskeletons is extremely expensive. Especially for developing countries where

Another important ethical concern is raised by highly expensive physical therapy devices. The new equipment developed for gait rehabilitation are a well-suited example. This equipment is unaffordable for public health institutions and affordable only in private medical practice. Therefore, may put in ethical dilemmas the patients and also the physiotherapists.

The patients mostly striving beyond their ability to access those services and the physiotherapists to have their professional work hampered, for not being able to provide full medical services. Some of these ethical challenges and dilemmas may be avoided by rules and cooperation of manufacturers, sellers, and national government.

\subsection{Inter-professional lack of communication and ethical concerns}

The analyze of the manufacturer's user manuals and physiotherapists professional associations guidelines for physiotherapy equipment use revealed that in some cases there are major discrepancies regarding the therapies contraindications.

Some of the associations and manufacturers permit using different types of electric agents like ultrasound on composite or metal implants, other don't.

It is quite a major mismatch in terms of contraindications regarding electrical implant carriers. Also from this perspective, certain associations and manufacturers allow the use of electrical agents to a patient with a pacemaker, but not in the chest area.

Most of the therapies are contraindicated to pregnant women, but not because of the physical agents' effects, but due to lack of studies in the area. Which is confirmed by some manufacturers in the user manuals [11-14]. Ethical reasoning does not mean a person should be confided into its personal boundaries in terms of thinking and application of codes of conduct or rules of moral behavior.

The ethical problem in this case appears when a patient might be harmed by a physiotherapist or a doctor, after the use of one these therapies, although the protocols of use were strictly followed. Whose responsibility is then? Is the medical professional to be blamed, the patentees, the bioengineers or the manufacturers?

And they often create ethical disputes and problems in some medical centers where physicians or physiotherapists disputed new knowledge about contraindications. There are cases where some professionals take into account the new permissiveness of technology, and others refuse to put them into practice for fear of injuring patients. Thus, the evolution and treatment of a patient may suffer or be gained by the absence or presence of a physical procedure that can cause it to recover faster or better.

Worldwide, in most countries, physiotherapists are independent medical professionals and they apply these medical procedures without being supervised by other medical specialists. In Romania and in several other countries there is an exception, due to physical medicine and rehabilitation doctors.

They make the medical prescription, they set the treatment parameters, the length and they decide what is indicated and what is contraindicated. This way they are also sharing the responsibilities with physical therapists, but shouldn't be shared also with medical engineers? The lack of communication between professionals like bioengineers, doctors, physiatrists and physical therapists can give rise to situations that endangered patient health.

Another problem that may arise from the use of physiotherapy equipment is related to the poor training of physiotherapists in using new equipment. In most cases, training is implemented by a sales representative of the company providing the equipment. And most of the time, these medical representatives do not have enough basic knowledge to get an appropriate training. Very often, not knowing answers to questions asked by the people they need to train. 


\subsection{Bioengineering ethical research and education}

The misconduct arose in research lately played an important role in ethical research frameworks. Federal or governmental agencies, academic institutions, professional association or professional communities have struggled to create specific rules for biomedical scientific research. The majority of research misconduct have emerged from the biomedical world and researches that prove that many medical pharmaceutical agencies that fund medical studies tend to overestimate the beneficial effect of their products [15].

Concerning physical therapy research, is very difficult to obtain accurate results because of two main factors: 1) the patient illness- which in most cases doesn't exist similarities that can provide scientific research results, and 2) the physical therapy manufacturers use different types of software design, with different electro physical parameters that sometimes are not disclosed in technical books [13-14].

The development of engineering process faces ethical issues related to principles like Justice, Beneficence, and Respect for Autonomy. There are many cases when bioengineers are somehow forced to accept to make compromise settings being vulnerable in front of the organizational management. The reflexive principium approaching for bioengineers is a useful tool for helping both specialists and students to face their ethical obligations, in the international context and cross-cultural bioengineering development. The conflict of interests that might arise in the engineering field is an ethical problem that needs to be discussed and solved because it affects also the engineers whose informed consent is not taken adequately and violating their autonomy.

The ethical education must be provided in a manner to determine the ethical reasoning skill. It is not enough just to offer information concerning what is ethic to do and what is not or knowledge about normative, descriptive and meta ethics. The process by which arises ethical reasoning skill needs more then information, needs applied ethics, problem case solving issues and the development of a specific ethical personal characteristic for the professionals in every field, but especially in the sciences. Ethical reasoning doesn't mean limiting personal thinking and application of codes of conduct or rules of moral behavior. Ethical reasoning is an It is a main component of cognitive and moral development process that must keep pace with the evolution of technology to prevent disasters affecting human life and to protect life, human dignity and human rights.

\section{Conclusions}

Physical therapy professionals, bioengineers, and doctors should communicate and create a team for offering quality medical services and respecting human rights and dignity.

A multidisciplinary team is needed for the physical therapy or electrotherapy research equipment development. There is a need also for a good communication channel between the physical therapist professionals and medical engineers for preventing the ethical dilemmas arising from the physical therapy equipment use.

There is a major need for ethical practice of problem based cases for biomedical engineers which perform their activities in the biomedical industry field. Codes of conduct and guidelines are very important instrument for ethical challenges raised in biomedical field, but they don't always approach each problem directly to equipment authors or engineers, one of these issues rarely delineated it concerns the authors' responsibility for controlling the translation process through the moment when the humans benefit from their creation. The ethical education must be provided in a manner to determine the ethical reasoning skill. This means the need for information, applied ethics, problem case solving issues and development of a specific ethical personal characteristic. Ethical reasoning doesn't mean limiting personal 
thinking and application of codes of conduct or rules of moral behavior. Ethical reasoning is the main component of cognitive and moral development process. It must keep pace with the evolution of technology, to prevent disasters affecting human life and to protect life, human dignity, and human rights.

As it regards the national government regulations it requires a multidisciplinary team, to realize a normative framework for the safe utilization of these devices. The framework must supply minimal technical and scientific devices requirements, include guidelines regarding the ethical implications and shared responsibility [16].

\section{References}

[1] J. Weiss, T. Pobre, L. Weiss, American Handbook of Physical Medicine \& Rehabilitation, Ed. New York: Oxford University Press, (2010).

[2] L., Rogozea, G.Sechel, A. Fleancu, Ethical aspects in bioengineering research., WSEAS International Conference. Proceedings. Mathematics and Computers in Science and Engineering, 139-143, (2009)

[3] W., Vela, L., Blanco, A., Gonzalez, J., Alococer, Major Trends In The Development Of Ankle Rehabilitation Devices, Dyna Rev.Fac.Nac.Minas, vol. 79, Dec(2012).

[4] L., Gloeckner, C., Fortunato, A., Pinkus, The Role of Professional Knowledge in CaseBased, Sci Eng. Ethics ,. 767-787,(2015)

[5] Aetna., Aetna Insurance Company. [Online], http://www.aetna.com/cpb/medical/data/300 399/0325.html, (2017)

[6] BTL, Shock Wave Users' Manual, (2008)

[7] N.B.M., Császár, N.B., Angstman, S., Milz, C.M., Sprecher, P., Kobel, M., Farhat, J.P., Furia, C., Schmitz, Radial Shock Wave Devices Generate Cavitation, Plos One, (2015.)

[8] World Health Organization, Medical Device Regulations-Global overview and guiding principles, Geneva, (2003)

[9] European Council, Council Directive 93/42/EEC of 14 June 1993 concerning medical devices, http://eur-lex.europa.eu/legal-content/EN/TXT/?uri=CELEX\%3A01993L004220071011

[10] B.A., Hagiu, The Physiotherapist Will Be Replaced By Robot? Sp Soc Int J Ph, vol. 16, 53-57, 2016

[11] Physiotherapy Canada. ELECTROPHYSICAL AGENTS-Contraindications and Precautions: An Evidence-Based Approach to Clinical Decision Making in Physical Therapy, Physiother Can, (2010)

[12] T., Watson. Electrotherapy, Contraindications, http://www.electrotherapy.org, (2007)

[13] Chatanooga, Djoglobal., User Manual, (2008)

[14] BTL-BTL 4000 Users' Manual, (2007)

[15] P., Herxheimer, A., Lexchin, J., Mansfield, P., Norris., Drug promotion what we know, what we have yet to learn-Reviews of materials in the WHO/HAI database on drug promotion WHO. WHO/EDM/PAR/, (2004)

[16] L., Rogozea, F., Leasu, A., Repanovici, M., Baritz, Ethics, robotics and medicine development, Proceedings of the 9th WSEAS international conference on Signal processing, robotics and automation,264-268, (2010) 